\title{
Runtime Support for Memory Adaptation in Scientific Applications via Local Disk and Remote Memory *
}

\author{
Chuan Yue $^{\dagger} \quad$ Richard T. Mills $s^{\ddagger} \quad$ Andreas Stathopoulos $^{\dagger} \quad$ Dimitrios Nikolopoulos $^{\dagger}$ \\ $\dagger$ Department of Computer Science $\quad$ National Center for Computational Sciences \\ College of William and Mary Oak Ridge National Laboratory \\ Williamsburg, VA 23187-8795 \\ Oak Ridge, TN 37831
}

\begin{abstract}
The ever increasing memory demands of many scientific applications and the complexity of today's shared computational resources still require the occasional use of virtual memory, network memory, or even out-of-core implementations, with well known drawbacks in performance and usability. In this paper, we present a general framework, based on our earlier MMLIB prototype [21], that enables fully customizable, memory malleability in a wide variety of scientific applications. We provide several necessary enhancements to the environment sensing capabilities of MMLIB and introduce a remote memory capability, based on MPI communication of cached memory blocks between 'compute nodes' and designated memory servers. We show experimental results from three important scientific applications that require the general MMLIB framework. Under constant memory pressure, we observe execution time improvements of factors between three and five over relying solely on the virtual memory system. With remote memory employed, these factors are even larger and significantly better than other, systemlevel remote memory implementations.
\end{abstract}

\section{Introduction}

Commoditization of memory chips has enabled unprecedented increases in the memory available on today's computers and at rapidly decreasing costs. Manufacturing and marketing factors, however, keep the costs disproportionally high for larger memory chips. Therefore,

\footnotetext{
*Work supported by the National Science Foundation (ITR/ACS0082094, ITR/AP-0112727, ITR/ACI-0312980, CAREER/CCF0346867) the Department of Energy (DOE-FG-02-05ER2568), a DOE computational sciences graduate fellowship, and the College of William and Mary. Part of this work was performed using SciClone cluster at the College of William and Mary which were enabled by grants from Sun Microsystems, the NSF, and Virginia's Commonwealth Technology Research Fund. Authors e-mail addresses: \{cyue,andreas,dsn\}@cs.wm.edu, rmills@ornl.gov.
}

although many shared computational resource pools or even large scale MPPs boast a large aggregate memory, only a small amount (relative to the high demands of many scientific applications) is available on individual processors. Moreover, available memory may vary temporally under multiprogramming. Sharing memory resources across processors is a difficult problem, particularly when applications cannot reserve sufficient memory for sufficiently long times. These realities pose tremendous problems in many memory demanding scientific applications.

To quantify the magnitude of the problem, we use a simple motivating example. We ran a parallel multigrid code to compute a three-dimensional potential field on four SMPs that our department maintains as a resource pool for computationally demanding jobs. Each SMP has 1 GB of memory. Since our code needed 860 MB per processor, we could run it using only one processor per node. While our code was running other users could run small jobs without interference. When a user attempted to launch Matlab to compute the QR decomposition of a large matrix on one of the processors in an SMP, the time per iteration in our multigrid code jumped from 14 to 472 seconds as virtual memory system thrashed. Most virtual memory systems would cause thrashing in this case, because their page replacement policies are not well suited for this type of scientific application.

Memory pressure can also be encountered on dedicated or space-shared COWs and MPPs. An important class of scientific applications runs a large number of sequential or low parallel degree jobs to explore a range of parameters. Typically, larger numbers of processors enable higher throughput computing. However, when memory is insufficient for individual jobs, such applications grind to a halt under virtual memory. On some MPPs, with small or no local disks, virtual memory is not adequate or even possible. Utilizing more processors per job to exploit larger aggregate memory may not be possible either, because the jobs are sequential or of limited parallel scalability. Application scientists often address these 
issues by implementing specialized out-of-core codes, and utilizing the parallel file systems that many MPPs employ. Besides the double performance penalty (data read from disk and propagated through the interconnection network), such codes often lack flexibility, performing $\mathrm{I} / \mathrm{O}$ even for data sets that could fit in memory.

Scenarios such as the above are one of the reasons for using batch schedulers, resource matchmakers, process migration and other techniques that warrant that each application will have enough memory to run without thrashing throughout its lifetime. These methods are not without problems. They may incur high waiting times for jobs, or high runtime migration overheads whenever there is resource contention. On certain platforms, such as shared-memory multiprocessors, some of these methods are not even applicable, as users may reserve more memory than their memory-per-CPU share [9]. A similar situation can occur if applications are allowed to use network RAM (NRAM) or other remote memory mechanisms [31]. Moreover, most remote memory research has been conducted at page-level granularity, which may incur unacceptably high latencies for page replacement [17].

The problem is equally hard at the sequential level. Page replacement policies of virtual memory systems usually target applications such as transaction processing that do not share the same memory access patterns as most scientific applications. In addition, high seek times during thrashing cannot be amortized by prefetching, as it is unreasonable to expect the virtual memory system to predict the locality and pattern of block accesses on disk. On the other hand, compiler or user-provided hints would require modifications to the system.

To tame these problems we have developed a runtime library, MMLIB (Memory Malleability library), that controls explicitly the DRAM allocations of specified large objects during the runtime of an application, thus enabling it to execute optimally under variable memory availability. MMLIB allows applications to use customized, application-specific memory management policies, running entirely at user-level. To achieve portability and performance, MMLIB blocks specified memory objects into panels and manages them through memory mapping. This provides programmers with a familiar interface that has no explicit I/O and can exploit the same common abstractions used to optimize code for memory hierarchies. Moreover, the advantages of running applications fully in-core are maintained, when enough memory is available. The library is designed for portability across operating systems and implemented in a nonintrusive manner.

In [21], we gave a proof of concept of MMLiB based on a simplified framework, and developed a parameterfree algorithm to accurately ascertain memory shortage and availability strictly at user-level. In this paper, we first provide a general framework that enables memory malleability in a variety of scientific applications, and enhance MMLIB's sensing capabilities to require no user input. Second, we introduce remote memory capability into MMLIB, based on MPI communication of panels between compute nodes and designated memory servers. Besides performance improvements on clusters with high speed networks, our flexible, user-level design enables a host of options such as multiple memory servers, and dynamic migration of panels between servers.

We see several benefits in this library-based approach for memory malleability. Injecting memory malleability into scientific applications allows them to run under memory pressure with degraded but acceptable efficiency, under a wider variety of execution conditions. Efficient memory adaptive applications can benefit both high-level batch schedulers, by letting them harness cycles from busy machines with idle memory, and operating system schedulers, by avoiding thrashing and thus securing continuous service to jobs. Also, as we show in this paper, the transparent design enables the library to implement remote memory when local disks are small or slower than fetching data from the network. MMLIB is an optimization tool for DRAM accesses at the low level of the memory hierarchy (disk/network), but it can coexist with and complement optimization tools for higher levels (memory/cache), enabling a unified approach to locality optimization in block-structured codes.

We present experimental results from three important scientific applications that we linked with MMLIB. Besides their importance for scientific computing, these applications stress different aspects of MMLIB and motivate the runtime optimizations presented in this work.

\section{Related work}

Interesting memory usage and availability patterns on multiprogrammed clusters of workstations has been pointed out in a quantitative study by Acharya and Setia [3]. They show that, on average, more than half of the memory of the clusters is available for intervals between 5 and 30 minutes, with shorter intervals for larger memory requests. The study did not investigate mechanisms and policies for exploiting idle memory or the impact of fluctuations of idle memory on application performance.

Batch schedulers for privately owned networks of workstations and grids, such as Condor-G [13] and the GrADS scheduling framework [10], use admission control schemes which schedule a job only on nodes with enough memory. This avoids thrashing at the cost of reduced utilization of memory and potentially higher job waiting times. Other coarse-grain approaches for avoiding thrashing include checkpointing and migration of 
jobs. However, such approaches are not generally aware of the performance characteristics or the execution state of the program [30].

Chang et.al. [8] have presented a user-level mechanism for constraining the resident set sizes of user programs within a fixed range of available memory. They assume that the program has an a-priori knowledge of lower and upper bounds of the required memory range. This work does not consider dynamic changes to memory availability, nor does it address the problem of customizing the memory allocation and replacement policy to the memory access pattern of the application-both central issues in our research.

An approach that addresses a dynamically changing environment for general applications has been developed by Brown and Mowry [7]. This approach integrates compiler analysis, operating system support, and a runtime layer to enable memory-intensive applications to effectively use paged virtual memory. The runtime layer makes the appropriate memory allocation decisions by processing hints on the predicted memory usage that the compiler inserted. Although the approach has shown some good results, it requires modifications to the operating system. In addition, applications with complex memory access patterns can cause significant difficulties in identifying appropriate release points.

Barve and Vitter [6] presented a theoretical framework for estimating the optimal performance that algorithms could achieve if they adapted to varying amounts of available memory. They did not discuss implementation details or how system adaptivity can be achieved. Pang et al. [27] presented a sorting algorithm that dynamically splits and merges the resident buffer to adapt to changes in the memory available to the DBMS. This is a simulation-based study that does not discuss any details of the adaptation interface.

Remote memory servers have been employed in multicomputers [15] for jobs that exceed the available memory per processor. They also enabled the implementation of diskless checkpointing [19], a fault-tolerance scheme which exploits the speed of the interconnection network to accelerate saving and restoring of program state.

The advent of high-throughput computing on shared computational resources has motivated the design of NRAM systems for clusters of workstations $[17,4,5$, $12,26]$. Real implementations of NRAM and memory servers $[5,15,11,31]$ extend the operating system paging algorithms and provide support for consistency and fault tolerance at the page level. Though performance improvements have been reported over disk-based virtual memory systems, the page level fine granularity still incurs significant overheads, and thrashing can still occur. Moreover, such implementations require substantial changes to the operating system.
Several researchers have utilized remote memory for implementing co-operative caching and prefetching in clustered web servers. Recently, Narravula et al. [18], exploit remote memory and direct remote DMA operations to improve utilization of aggregate distributed caches in a co-operative caching environment. Our work differs in that it employs remote memory in an applicationcontrolled execution environment.

Koussih et al. [16] describe a user-level, remote memory management system called Dodo. Based on a Condor-like philosophy, Dodo harvests unused memory from idle workstations. It provides allocation and management of fine-grained remote memory objects, as well as user-defined replacement policies, but it does not include adaptation mechanisms for dynamically varying RAM, and cannot apply to local disks, or to remote memory servers that are not idle.

In [25], Nikolopoulos presented an adaptive scheduling scheme for alleviating memory pressure on multiprogrammed COWs, while co-ordinating the scheduling of the communicating threads of parallel jobs. That scheme required modifications to the operating system. In [24], the same author suggested the use of dynamic memory mapping for controlling the resident set of a program within a range of available physical memory. The algorithm operated at page-level granularity. In [22], two of the authors of this paper followed an application-level approach that avoided thrashing of an eigenvalue solver, by having the node under memory pressure recede its computation during the most computationally intensive phase, hopefully speeding the completion of competing jobs.

MMLIB bares similarities with SHMOD (SharedMemory on Disk) [32], an application-level asynchronous remote I/O library, which enables effective remote disk space usage, continuous application-level checkpointing and out-of-core execution. SHMOD is designed to support specifically a class of hydrodynamics applications and uses coarse-grain panels (called things in SHMOD's terminology), allocated and managed transparently across local and remote disks in a cluster of workstations. MMLIB differs in that it utilizes remote DRAM instead of remote disks for faster retrieval of panels.

\section{User-level adaptation}

Application-level approaches are sometimes received with caution because of increased developer involvement. However, to exploit higher memory hierarchies, developers of scientific applications already block the accesses to the data or use libraries where such careful blocking is provided. Blocking for memory access is performed at a much larger granularity and thus complementary to cache 
access. Based on this, our approach in [21] considered the largest data object partitioned into $P$ blocks, which in out-of-core literature are often called panels, and operated as follows:

for $\mathrm{i}=1: P$

Get panel $p_{\text {pattern }(i)}$ from lower level memory

Work with $p_{\text {pattern(i) }}$

Most scientific applications consist of code segments that can be described in this familiar to developers format. As long as the "get panel" encapsulates the memory management functionality, no code restructuring is ever needed.

On a dedicated workstation one can easily select between an in-core or an out-of-core algorithm and data structure according to the size of the problem. On a nondedicated system though, the algorithm should adapt to memory variability, running as fast as an in-core algorithm if there is enough memory to store its entire data set, or utilizing the available memory to cache as many panels as possible. Based on memory mapped I/O, we provided a framework and supporting library for modifying codes for memory adaptivity that are portable to many applications and operating systems. Memory mapping has several advantages over conventional I/O because it avoids write-outs to swap space of read-only panels, integrates data access from memory and disk, allows for fine tuning of the panel size to hide disk latencies and facilitates an implementation of various cache replacement policies.

To get a new panel, the application calls a function from our library that also controls the number of panels kept in-core. At this point, the function has three choices: it can increase or decrease the number of in-core panels if additional, or respectively less, memory is available; or it can sustain the number of in-core panels if no change in memory availability is detected. The policy for selecting panels to evict is user defined as only the application has full knowledge of the access pattern.

Critical to this functionality is that our library be able to detect memory shortage and availability. However, the amount of total available memory is a global system information that few systems provide, and even then, it is expensive and with no consistent semantics. In [21], we developed an algorithm which relies only on measurements of the program's resident set size (RSS), a widely portable and local information. Memory shortage is inferred from a decrease in a program's RSS that occurs without any unmapping on the part of the program. Memory surplus is detected using a "probing" approach in which the availability of a quantity of memory is determined by attempting to use it and seeing if it can be maintained in the resident set. The algorithm is parameterfree, expecting only an estimate of the memory requirements of the program, excluding the managed panels. We

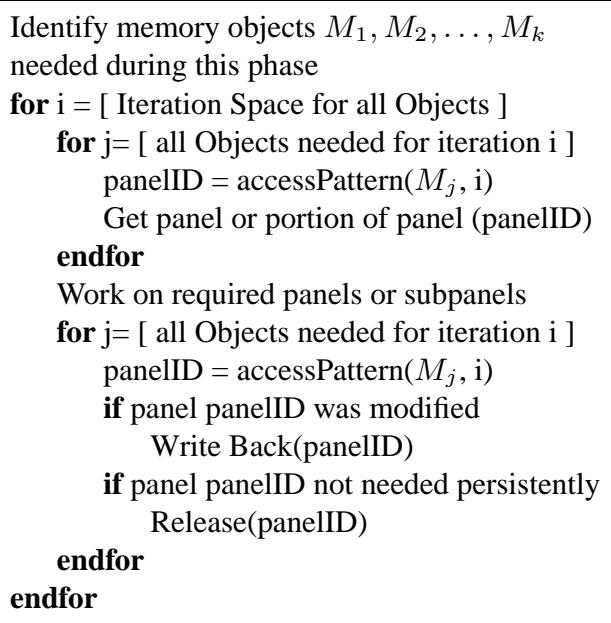

Figure 1. Extended framework modeling the memory access needs of a wide variety of scientific applications.

call the size of this non-managed memory, static memory (sRSS). The algorithm detects memory availability, by probing the system at dynamically selected time intervals, attempting to increase memory usage one panel at a time. For the effectiveness of this algorithm in identifying the available memory, the reader is referred to [21].

\subsection{A general framework}

The above simplified framework is limited to a class of applications with repeated, exhaustive passes through one, read-only object. We have developed a more comprehensive framework that captures characteristics from a much larger class of applications. Scientific applications often work on many large memory objects at a time, with various access patterns for each object, sometimes working persistently on one panel, while other panels are only partially accessed, or even modified. This extended framework of applications is shown in Figure 1.

Figure 1 depicts only one computation phase, which is repeated several times during the lifetime of the program. A computation phase denotes a thematic beginning and end of some computation, e.g., one iteration of the CG method or the two innermost of three nested loops. In this phase, a small number of memory objects are accessed (e.g., the matrix and the preconditioner in the CG algorithm), as their sheer size limits their number. In contrast to the previous simplified framework, we do not assume a sequential pass through all the panels of an object, although this is often the case in practice. In this context, a full sweep denotes a completion of the phase.

For each iteration of the computation phase, certain panels from certain memory objects need to be fetched, 
worked upon, and possibly written back. The iteration space in the current computation phase, the objects needed for the current iteration, and the access patterns for panels depend on the algorithm and can be described by the programmer. Finally, our new framework allows memory objects to fluctuate in size between different computation phases.

We have extended MMLIB to implement this general framework, hiding all bookkeeping and adaptation decisions from the user. The user interface that enables this transparency and a detailed discussion of the technical issues involved are described in detail in [20].

\subsection{Estimating static memory size}

Our memory adaptation algorithm in [21] assumes that the program has an accurate estimate of the size of its static memory, i.e., memory not associated with managed objects. This is needed for calculating how much of the RSS belongs to the mapped objects. However, this size may not be easily computed or even available to the program if large enough static memory is allocated within linked, third party libraries. Moreover, for some programs the static memory may fluctuate between sweeps of the computation phase. A more serious problem arises when the static memory is not accessed during the computation phase. Under memory pressure, most operating systems consider the static memory least recently used and slowly swap it out of DRAM. This causes a false detection of memory shortage, and the unmapping of as many panels as the size of the swapped static memory.

An elegant solution to this problem relies on a system call named mincore () for most Unix systems and VirtualQuery () for Windows. The call allows a process to obtain information about which pages from a certain memory segment are resident in core. Because MMLIB can only ascertain residency of its managed memory objects, it uses mincore () to compute the actual resident size of the managed panels, which is exactly what our algorithm needs. Obviously, the use of this technique at every get_panel is prohibitive because of the overhead associated with checking the pages of all mapped panels. We follow a more feasible, yet equally effective strategy. We measure the residency of all panels (mRSS) in the beginning of a new computational phase, and derive an accurate estimate of the static memory: sRSS $=$ RSS - mRSS, with RSS obtained from the system. As long as no memory shortage is detected, we use sRSS during the computation phase. Otherwise, we recompute mRSS and sRSS to make sure we do not unnecessarily unmap panels. Since unmapping is not a frequent occurrence the overall mincore overhead is tiny, especially compared to the slow down the code experiences when unmapping is required.

\subsection{A most-missing eviction policy}

One of the design goals of MMLIB is to preempt the virtual memory system paging policy by holding the RSS of the application below the level at which the system will begin to swap out the pages of the application. Under increasing memory pressure, the paging algorithm of the system could be antagonistic by paging out data that MMLIB tries to keep resident, thus causing unnecessary additional memory shortage. In this case, it may be beneficial to "concede defeat" and limit our losses by evicting those panels that have had most of their pages swapped out, rather than evicting according to our policy, say MRU. The rationale is that if the OS has evicted LRU pages, these will have to be reloaded either way, so we might as well evict the corresponding panels. Evicting MRU panels may make things worse because we will have to load the swapped out LRU pages as well as the MRU panels that we evicted.

The mincore functionality we described above facilitates the implementation of this "most missing" policy. This policy is not at odds with the user specified policy because it is only applied when memory shortage is detected, which is when the antagonism with the system policy can occur. Under constant or increasing memory availability the user policy is in effect. Preliminary results in section 5 show clear advantages with this policy.

\section{Remote memory extension}

Despite a dramatic increase in disk storage capacities, improvements in disk latencies have lagged significantly behind those of interconnection networks. Hence, remote virtual memory has often been suggested [17]. The argument is strengthened by work in $[3,2,16]$ showing that there is significant benefit to harvesting the ample idle memory in computing clusters for data-intensive applications. The argument is imposing on MPPs, where parallel I/O must pass also through the interconnection network.

The general MMLIB framework in Figure 1 lends itself naturally to a remote memory extension. The key modification is that instead of memory mapping a new panel from the corresponding file on disk, we allocate space for it and request it from a remote memory server. This server stores and keeps track of unmapped panels in its memory, while handling the mapping requests. In implementing this extension we had to address several design issues.

First, we chose MPI for the communication between processors, because it is a widely portable interface that users are also familiar with. Also, because MMLIB works entirely at user-level, we need the user to be able to designate which processors will play the role of remote memory servers. This is deliberately unlike the Dodo or 
Condor systems that try to scavenge idle memory and cycles in COWs. The long experience of some of the authors in scientific programming suggests that users are empowered, not burdened by exercising this control. The downside of using MPI is that the user must compile and run sequential programs with MPI. All other MPI set up and communications are handled internally in MMLIB. For parallel programs there is no additional burden to the user. In the future, and if experience deems it necessary, a more transparent communication mechanism can be implemented with minimal change to MMLIB. Nevertheless, our implementation is practically transparent, and it has provided a proof of concept for this functionality.

Second, because each panel is associated with a particular remote server, the executing process knows where to request it from and there is no need for consistency maintenance between replicas of panels. This allows the panels of one memory object to be kept on a number of servers. At the same time each server may be storing and handling panels from many objects, and possibly from many processors. Because of the large granularity, there is only a small number of panels, so the additional bookkeeping is trivial. This flexible design, which is reminiscent of home-based shared virtual memory research [14], enables a load balancing act between servers, that can migrate panels completely independently from the execution nodes. As long as the server pointer of each unmapped panel is updated in the corresponding managed memory object, execution nodes know where to direct their next request. An exploration of the many possibilities that arise from this design as well as more fine-grain consistency models is beyond the scope of this paper.

Third, the memory that will hold a remotely fetched panel does not have to be allocated with named memory mapping. Named memory mapping was important in the original MMLIB as it was used to read a panel implicitly from a disk file, and because of that file it could avoid writing to the swap device under memory pressure. With remote memory, the existence of a file image for each panel is not required. We have explored the question of which allocation mechanism among malloc (), named mmap (), and anonymous mmap () provides the most benefits in performance and flexibility. Our experimental testbed and results, shown in the following section, yield anonymous mmap () as the best choice. In principle, memory mapping should be preferred because it permits the use of mincore () by MMLIB to compute the static memory size, and thus provide accurate sensing measurements for adaptation. On some operating systems malloc () is not implemented on top of mmap (), and thus does not permit the use of mincore () .

An outline of the remote memory algorithm follows. Initially, the memory server(s) load all the (initially unmapped) panels of all objects of the applica- tion into their memory. When a working process issues a get_panel (mms, p), and the panel is not in memory (mapped), MMLIB sends a request to the appropriate server holding panel $\mathrm{p}$ of the object mms. If no panel is to be unmapped, MMLIB allocates the appropriate memory space and issues an MPI_Recv. If a panel, $q$, is to be unmapped, MMLIB figures out the server to send it to, and initiates an MPI_Send. When the send returns, this space of q can be reused to store the incoming panel $\mathrm{p}$, so MMLIB simply issues an MPI_Recv. We should point out that if an object is designated as read-only, its panels need not be sent to the memory server when unmapped, provided that the server keeps all panels in its memory. Finally, the MMLIB framework retains all of its adaptivity to external memory pressure when our remote memory implementation is used in place of disk I/O.

\section{Experiments}

First we describe three applications that we modified to use MMLIB; their special characteristics require our extended framework and optimizations and cannot be implemented using the simple framework of [21]. Second, we present experiments with these applications under constant and variable memory pressure. Third, we explore experimentally the question posed in the previous section about the most appropriate allocation mechanism for remote memory. Finally we demonstrate the power of remote memory in MMLIB using the CG application.

\subsection{The applications}

The first application is the conjugate gradient (CG) linear system solver provided in SPARSKIT [28]. Each iteration of CG requires one sparse matrix-vector multiplication and a few inner products and vector updates. Our only managed object is the coefficient matrix, as it poses the bulk of the memory demands of the program, and is broken into 40 panels. CG also has a sizable amount of static memory for six work vectors. For MMLIB to work, this size must be known. In [21], we hard coded the size of this static memory. Here, we let MMLIB detect it dynamically.

The second application is a modified Gram-Schmidt (MGS) orthogonalization procedure. A memory demanding application of MGS stems from materials science, where Krylov eigensolvers are used to find about 500-2000 eigenvectors for an eigenvalue problem of dimension on the order of one million [29]. Our code simulates a Krylov eigensolver except that it generates the recurrence vector at random, not through matrix vector multiplication. This vector is orthogonalized against previously generated vectors and then appended to them. Only these vectors need to be managed by MMLIB. In 
our experiment, we use one panel per vector for a total of 30 vectors, each of 3,500,000 doubles (80 MB total). This test is possible only through our new MMLIB, as the size of the managed object varies at runtime, and multiple panels are active simultaneously.

The third application is an implementation of the Ising model, which is used to model magnetism in ferromagnetic material, liquid-gas transition, and other similar physical processes. Considering a two-dimensional lattice of atoms, each of which has a spin of either up or down, the code runs a Monte-Carlo simulation to generate a series of configurations that represent thermal equilibrium. The memory accesses are based on a simple 5point stencil computation. For each iteration the code sweeps the lattice and tests whether to flip the spin of each lattice site. The flip is accepted, if it causes a negative potential energy change, $\Delta E$. Otherwise, the spin flips with a probability equal to $\exp \frac{-\Delta E}{k T}$, where $k$ is the Boltzman constant and $T$ the ambient temperature. The higher the $T$, the more spins are flipped. In computational terms, $T$ determines the frequency of writes to the lattice sites at every iteration. The memory-adaptive version partitions the lattice row-wise into 40 panels. To calculate the energy change at panel boundaries, the code needs the last row of the above neighboring panel and the first row of the below neighboring panel. The new MMLIB framework is needed for panel write backs with variable frequency, and multiple active panels.

In all three applications the panel replacement policy is MRU, but there is also use of persistent (MGS) and neighboring (Ising) panels.

\subsection{Adaptation results}

Figure 2 includes one graph per application, each showing the performance of three versions of that application under constant memory pressure. Each point in the charts shows the execution time of one version of the application when run against a dummy job that occupies a fixed amount of physical memory, using the mlock () system call to pin its pages in-core. For each application we test a conventional in-core version (blue top curve), a memory-adaptive version using MMLIB (red lower curve), and an ideal version (green lowest curve) in which the application fixes the number of panels cached at an optimal value provided by an oracle. The charts show the performance degradation of the applications under increasing levels of memory pressure. In all three applications, the memory-adaptive implementation performs consistently and significantly better than the conventional in-core implementation. Additionally, the performance of the adaptive code is very close to the ideal-case performance, without any advance knowledge of memory availability and static memory size, and re-
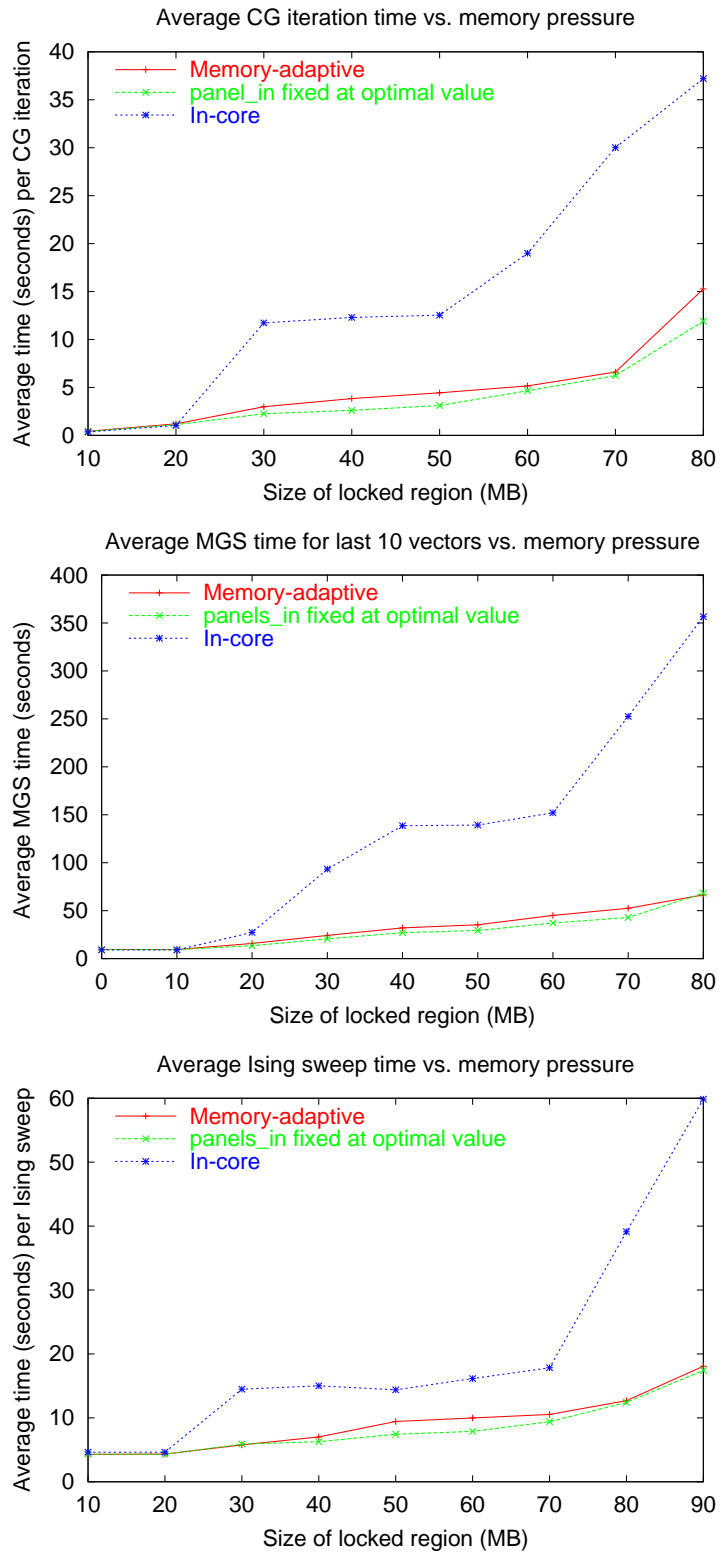

Figure 2. Performance under constant memory pressure. The top chart shows the average time per iteration of CG with a 70 MB matrix, which requires a total of 81 MB of RAM including memory for the work vectors. The middle chart shows the time to orthogonalize via modified Gram-Schmidt the last 10 vectors of a $\mathbf{3 0}$ vector set. Approximately $80 \mathrm{MB}$ are required to store all $\mathbf{3 0}$ vectors. The bottom chart shows the time required for an Ising code to sweep through a $70 \mathrm{MB}$ lattice. All experiments were conducted on a Linux 2.4.22-xfs system with 128 MB of RAM, some of which is shared with the video subsystem. 
gardless of the number of active panels (whether readonly or read/write).

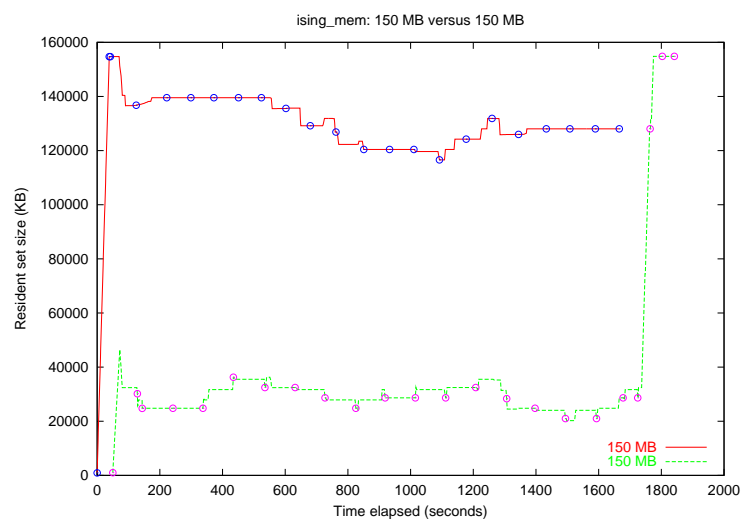

Figure 3. Running adaptive versus adaptive jobs. RSS vs. time for two equal-sized (150 MB) memory-adaptive Ising jobs. The second job starts $\mathbf{3 0}$ seconds after the first job. The experiment is run on a Sun Ultra 5 with 256 MB of RAM, under Solaris 9.

The litmus test for MMLIB is when multiple instances of applications employing the library are able to coexist on a machine without thrashing the memory system. Figure 3 shows the resident set size (RSS) over time for two instances of the memory adaptive Ising code running simultaneously on a Sun Ultra 5 node. After the job that starts first has completed at least one sweep through the lattice, the second job starts. Both jobs have $150 \mathrm{MB}$ requirements, but memory pressure varies temporally. The circles in the curves denote the beginning of lattice sweeps. Distances between consecutive circles along a curve indicate the time of each sweep.

The results show that the two adaptive codes run together harmoniously without constantly evicting each other from memory and the jobs reach an equilibrium where system memory utilization is high and throughput is sustained without thrashing. The system does not allow more than about $170 \mathrm{MB}$ for all jobs, and it tends to favor the application that starts first. A similar phenomenon was observed in Linux. We emphasize that the intricacies of the memory allocation policy of the OS are orthogonal to the policies of MMLIB. MMLIB allows jobs to utilize as efficiently as possible the available memory, not to claim more memory than what is given to each application by the OS.

Figure 4 shows the benefits of our proposed "mostmissing" eviction policy. After external memory pressure starts, the job that uses strictly the MRU policy has highly variable performance initially because the evicted MRU panels usually do not coincide with the system evicted

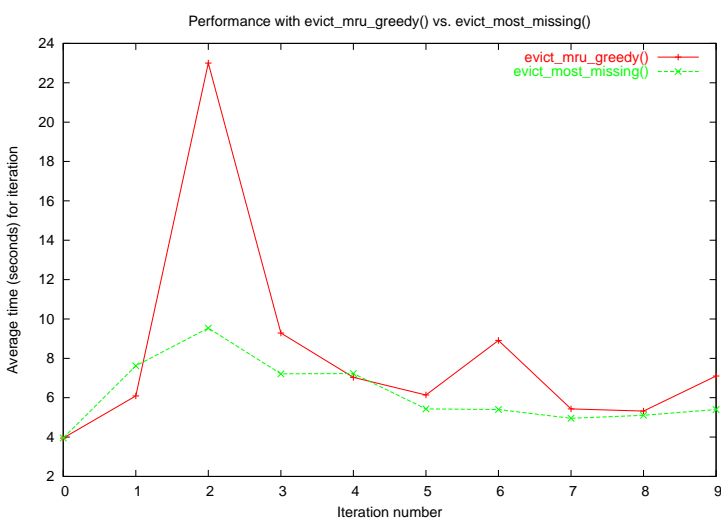

Figure 4. Benefits of evicting partially swapped out panels. The chart shows the times for the first 10 sweeps of a memory-adaptive Ising job running with a $60 \mathrm{MB}$ lattice on a Linux machine with 128 MB of RAM. After 12 seconds, a dummy job that uses $60 \mathrm{MB}$ of memory begins. The job that evicts panels with the largest number of missing pages, labeled evict most_missing, has lower and less variable times than the original MRU replacement, labeled evict_mru_greedy.

pages. The most missing policy is much better during that phase. After the algorithm adapts to the available memory the most missing policy reverts automatically to MRU.

Because all three of the applications we have tested employ memory access patterns for which MRU replacement is appropriate (and are thus very poorly served by the LRU-like algorithms employed by virtual memory systems), one might wonder if all of the performance gains provided by MMLIB are attributable to the MRU replacement that it enables. To test this notion, we ran the MMLIB-enabled CG under memory pressure and instructed MMLIB to use LRU replacement. Figure 5 compares the performance of CG using the wrong (LRU) replacement policy with the CG correctly employing MRU. The version using LRU replacement performs significantly worse, requiring roughly twice the amount of time required by the MRU version to perform one iteration. However, when compared to the performance of in-core CG under memory pressure, the code using the wrong replacement policy still performs iterations in roughly half the time of in-core CG at lower levels of memory pressure, and at higher levels performs even better. As discussed in the designing goals of MMLIB, the benefits in this case come strictly from the large granularity of panel access, as opposed to the page-level granularity of the virtual memory system, and from avoiding thrashing. 


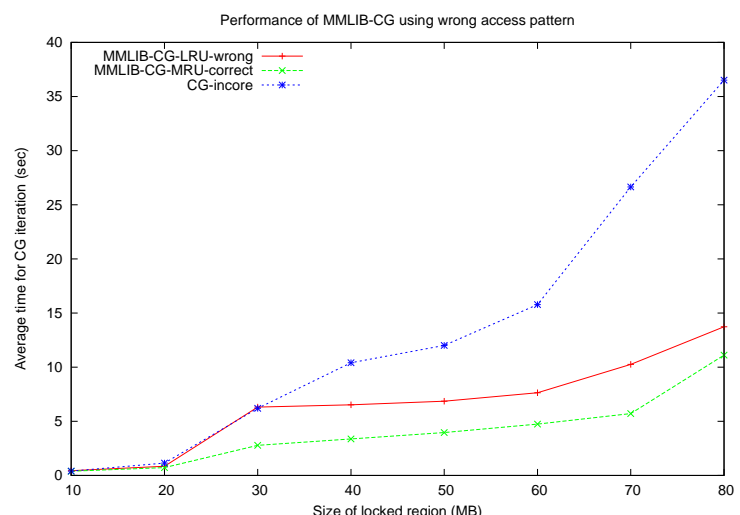

Figure 5. Performance of memory-adaptive CG versus memory pressure when using the wrong panel replacement policy, which is depicted by the curve labeled MMLIB-CG-LRU-wrong. The appropriate replacement policy is MRU, but LRU is used instead; any observed performance gains are not due to the ability of MMLIB to allow application-specific replacement. For comparison, the performance of memory-adaptive correctly employing MRU is depicted by the curve labeled MMLIB-CG-MRU-correct. Jobs run under static memory pressure provided by locking a region of memory in-core on a Linux 2.4 system with 128 MB of RAM.

\subsection{Remote memory results}

The remote memory experiments are conducted on the SciClone cluster [1] at William \& Mary. All programs are linked with the MPICH_GM package and the communications are routed via a Myrinet 1280 switch. We use dual-cpu Sun Ultra 60 workstations at $360 \mathrm{MHz}$ with 512MB memory, running Solaris 9.

\subsubsection{Microbenchmark results}

These experiments help us understand the effect of various allocation schemes (malloc, named mmap, anonymous mmap) on the MPI Recv performance, under various levels of memory pressure. Figure 6 shows three graphs corresponding to the three methods for allocating the receiving block of the MPI_Recv call. Each graph contains six curves plotting the perceived MPI_Recv bandwidth for six different total 'buffers' that the MPI Recv tries to fill by receiving 'Block Size' bytes at a time. This microbenchmark simulates an MMLIB process that uses remote memory to bring each one of the panels (of 'Block Size' each) of a memory object (of 'buffer' size). On the same node with the receiving process, there is a competing process reading a $300 \mathrm{MB}$ file
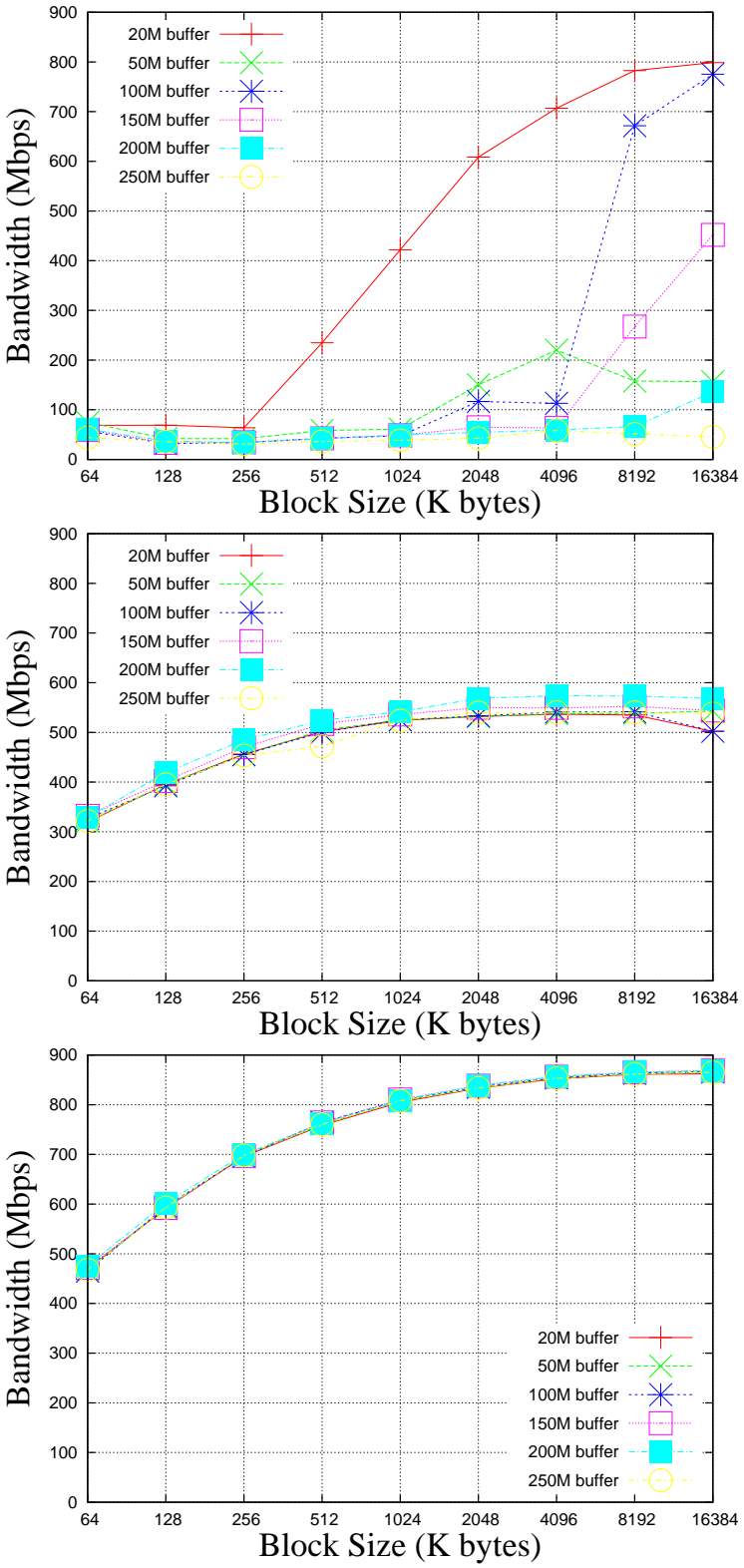

Figure 6. MPI_Recv perceived bandwidth microbenchmark results. The charts show the perceived MPI_Recv bandwidth vs. receiving block size for six different total buffer sizes. The receiving block is allocated using named mmap () (top chart), anonymous mmap () (middle chart), or malloc () (bottom chart). 
from the local disk. The larger the total 'buffer' size, the more severe the memory pressure on the node. The remote memory server process is always ready to send the requested data.

The top chart suggests that named mapping is the worst choice for allocating MPI Recv buffers, especially under heavy memory pressure, which is exactly when MMLIB is needed. In fact when the receiving block is small, performance is bad even without memory pressure (e.g., all curves for block size of $256 \mathrm{~KB}$ ). With large block sizes bandwidth increases but only when the total 'buffer' does not cause memory pressure (e.g., the 20MB 'buffer' curve). A reason for this is that receiving a remote panel causes a write-out to its backing store on the local disk, even though the two may be identical.

For both the middle and bottom charts, all six curves are very similar, suggesting that allocation using anonymous mmap() or malloc() is not very sensitive to memory pressure. The MPI Recv bandwidth performance of using malloc () for memory allocation is better than that of using anonymous mmap (). As no other processes were using the network during the experiments, the perceived differences in bandwidth must be due to different mechanisms of Solaris 9 for copying memory from system to user space.

Although these microbenchmarks suggest malloc() as the allocator of choice for implementing remote memory, experiments with CG favor anonymous mapping, with malloc() demonstrating unpredictable behavior.

\subsubsection{CG application results}

To demonstrate the remote memory capability of MMLIB, we performed two sets of experiments with the CG application on the same computing platform as in microbenchmark experiments.

In the first set, the MMLIB enabled CG application runs on one local node. It works on a 200MB matrix, which is equally partitioned into 20 panels. We create various levels of memory pressure on the local node by running in addition the in-core $\mathrm{CG}$ application (without MMLIB) with $300 \mathrm{MB}, 150 \mathrm{MB}$, and $100 \mathrm{MB}$ memory requirements. The experimental results are shown in Table 1. There are two rows of data for each of the three memory allocation methods. In the first row, we run the MMLIB CG without remote memory; in the second row, we run MMLIB CG with remote memory capability.

First, we see that under named mmap(), performance for remote mode is inferior to local mode, while under anonymous mmap () mode and malloc(), remote mode is obviously superior to local mode, especially when memory pressure is severe. The results also confirm the microbenchmark observations that named mmap () is the wrong choice for remote memory.
In contrast to the microbenchmark results, however, remote mode performance is better under anonymous mmap () than under malloc(). There are two reasons for this. The most important reason is that on Solaris 9 malloc () extends the data segment by calling brk(), rather than by calling mmap(). Because MMLIB issues a series of malloc() and free() calls, especially when there is heavy memory pressure, the unmapped panels may not be readily available for use by the system. This causes the runtime scheduler to think that there is not enough memory and thus to allocate less resources to the executing process. In our experiments on the dual cpu Suns, we noticed that the Solaris scheduler gave less than $25 \%$ cpu time to the application in malloc () mode, while it gave close to $50 \%$ cpu time to the one in anonymous mmap () mode. Interestingly, the local MMLIB implementation without remote memory demonstrates even a worse behavior, suggesting that, on Solaris, the use of malloced segments should be avoided for highly dynamic I/O cases. The second reason is that the mincore () system call does not work on memory segments allocated by malloc (). Therefore, MMLIB cannot obtain accurate estimates of the static memory to adapt to memory variabilities.

In the second set of the experiments, we let two MMLIB CG applications run against each other to measure the performance advantages of using remote memory over local disk in a completely dynamic setting. Both MMLIB CG applications use anonymous mmap () to allocate memory and work on different matrices of equal size. Each matrix is equally partitioned into $10 \mathrm{MB}$ panels. The experimental results are shown in Table 2. When remote memory instead of local disk is used, the total wall-clock time of the two MMLIB CG applications is always reduced. Especially when the overall memory pressure is severe such as the $300 \mathrm{MB}$ matrix and $250 \mathrm{MB}$ matrix cases (the overall memory requirement for data is $600 \mathrm{MB}$ and 500MB), the wall-clock time reduction can be $22.4 \%$ and $28.7 \%$ respectively.

We emphasize that these improvements are on top of the improvements provided by the local disk MMLIB over the simple use of virtual memory. Considering also the improvements from Figure 2, our remote memory library improves local virtual memory performance by a factor of between four and seven. This compares favorably with factors of two or three reported in other remote memory research [16].

\section{Conclusions}

We presented a general framework and supporting library that allows scientific applications to automatically manage their memory requirements at runtime, thus executing optimally under variable memory availability. 
Wall-clock time for MMLIB CG running against in-core CG

\begin{tabular}{|l|cccc|}
\hline \multicolumn{1}{|c|}{ memory pressure } & \multirow{2}{*}{ 300MB } & $150 \mathrm{MB}$ & \multirow{2}{*}{$100 \mathrm{MB}$} & \multirow{2}{*}{ Without Pressure } \\
\hline mamed mmap () local & 388.097 & 326.642 & 309.365 & 285.135 \\
named mmap () remote & 484.34 & 472.831 & 371.277 & 289.617 \\
\hline anonymous mmap () local & 541.005 & 367.357 & 317.726 & 294.406 \\
anonymous mmap () remote & 379.114 & 360.516 & 317.756 & 293.213 \\
\hline malloc () local & 1325.485 & 1023.782 & 1059.640 & 1050.507 \\
malloc () remote & 534.229 & 504.043 & 475.662 & 462.117 \\
\hline
\end{tabular}

Table 1. This table shows the wall-clock time for MMLIB CG running against in-core CG, in six modes corresponding to the six rows in the table. CG works on a 200MB matrix on local node. Memory pressure is created by in-core CG running on local node. Without Pressure means in-core CG is not running.

Wall-clock time for MMLIB CG running against MMLIB CG

\begin{tabular}{|c|c|c|c|c|c|}
\hline $\begin{array}{ll}\text { processes } & \text { matrix size }\end{array}$ & 300MB & $250 \mathrm{MB}$ & $200 \mathrm{MB}$ & 150MB & 100MB \\
\hline$c g 1$ local & 1496.700 & 1116.355 & 413.500 & 265.929 & 181.252 \\
\hline$c g 2$ local & 1015.495 & 755.859 & 638.448 & 266.697 & 185.796 \\
\hline Total local & 2512.195 & 1872.214 & 1051.948 & 532.626 & 367.048 \\
\hline$c g 1$ remote & 1139.011 & 815.240 & 415.056 & 266.049 & 158.954 \\
\hline$c g 2$ remote & 809.446 & 519.031 & 603.588 & 252.822 & 157.289 \\
\hline Total remote & 1948.457 & 1334.271 & 1018.644 & 518.871 & 316.243 \\
\hline Remote time reduction over local & $22.4 \%$ & $28.7 \%$ & $3.16 \%$ & $2.58 \%$ & $13.8 \%$ \\
\hline
\end{tabular}

Table 2. This table shows the wall-clock time for MMLIB CG running against MMLIB CG. Both MMLIB CG applications use anonymous mmap () to allocate memory. The first three rows show the results when local disk is used by both MMLIB CG applications. The following three rows show the results when remote memory is used by both MMLIB CG applications. The last row shows the total wall-clock time reduction for remote over local mode.

The library is highly transparent, requiring minimal code modifications and only at a large granularity level.

This paper extends our previous simplified framework and adaptation algorithm for memory malleability with the following key functionalities: (a) multiple and simultaneous read/write memory objects, active panels, and access patterns, (b) automatic and accurate estimation of the size of the non-managed memory, and (c) application level remote memory capability.

We showed how each of the new functionalities (a) and (b) were necessary in implementing three common scientific applications, and how (c) has significant performance advantages over system-level remote memory. Moreover, the remote memory functionality has opened a host of new possibilities for load and memory balancing in COWs and MPPs, that will be explored in future research. Our experimental results with MMLIB, have confirmed its adaptivity and near optimality in performance.

\section{References}

[1] SciClone cluster project at the College of William and
Mary. 2005.

[2] A. Acharya, G. Edjlali, and J. Saltz. The utility of exploiting idle workstations for parallel computation. volume 25 , pages 225-234, 1997.

[3] A. Acharya and S. Setia. Availability and Utility of Idle Memory in Workstation Clusters. In Proc. of the 1999 ACM SIGMETRICS Joint International Conference on Measurement and Modeling of Computer Systems (SIGMETRICS'99), pages 35-46, Atlanta, Georgia, May 1999.

[4] A. Acharya, M. Uysal, R. Bennett, A. Mendelson, M. Beynon, J. Hollingsworth, J. Saltz, and A. Sussman. Tuning the Performance of I/O Intensive Parallel Applications. In Proc. of the Fourth Workshop on Input/Output in Parallel and Distributed Systems (IOPADS'96), pages 15-27, Philadelphia, PA, May 1996.

[5] A. Barak and A. Braverman. Memory Ushering in a Scalable Computing Cluster. Journal of Microprocessors and Microsystems, 22(3-4):175-182, Aug. 1998.

[6] R. D. Barve and J. S. Vitter. A theoretical framework for memory-adaptive algorithms. In IEEE Symposium on Foundations of Computer Science, pages 273-284, 1999.

[7] A. D. Brown and T. C. Mowry. Taming the memory hogs: Using compiler-inserted releases to manage phys- 
ical memory intelligently. In Proceedings of the 4th Symposium on Operating Systems Design and Implementation (OSDI-00), pages 31-44, 2000.

[8] F. Chang, A. Itzkovitz, and V. Karamcheti. User-Level Resource Constrained Sandboxing. In Proc. of the 4th USENIX Windows Systems Symposium, pages 25-36, Seattle, WA, Aug. 2000.

[9] S. Chiang and M. Vernon. Characteristics of a Large Shared Memory Production Workload. In Proc. 7th Workshop on Job Scheduling Strategies for Parallel Processing (JSSPP'2001), Lecture Notes in Computer Science, Vol. 2221, pages 159-187, Cambridge, MA, June.

[10] H. Dail, H. Casanova, and F. Berman. A Decoupled Scheduling Approach for the GrADS Program Development Environment. In Proc. of the IEEE/ACM Supercomputing'02: High Performance Networking and Computing Conference (SC'02), Baltimore, MD, Nov. 2002.

[11] M. Feeley, W. Morgan, F. Pighin, A. Karlin, H. Levy, , and $\mathrm{C}$. Thekkat. Implementing global memory management in a workstation cluster. In 15th ACM Symposium on Operating Systems Principles(SOSP-15), pages 201212, 1995.

[12] M. Flouris and E. Markatos. Network RAM. In High Performance Cluster Computing, pages 383-408. Prentice Hall, 1999.

[13] J. Frey, T. Tannenbaum, M. Livny, I. Foster, and S. Tuecke. Condor-G: A Computation Management Agent for Multi-Institutional Grids. In Proc. of the 10th IEEE International Symposium on High Performance Distributed Computing (HPDC-10), pages 55-63, San Francisco, California, Aug. 2001.

[14] L. Iftode. Home-Based Shared Virtual Memory. PhD thesis, Princeton University, June 1998.

[15] L. Iftode, K. Petersen, and K. Li. Memory Servers for Multicomputers. In Proc. of the IEEE 1993 Spring Conference on Computers and Communications (COMPCON'93), pages 538-547, Feb. 1993.

[16] S. Koussih, A. Acharya, and S. Setia. Dodo: A user-level system for exploiting idle memory in workstation clusters. In HPDC, 1999.

[17] E. P. Markatos and G. Dramitinos. Implementation of a reliable remote memory pager. In USENIX Annual Technical Conference, pages 177-190, 1996.

[18] S. Narravula, H. W. Jin, K. Vaidyanathan and D. K. Panda. Designing Efficient Cooperative Caching Schemes for Multi-Tier Data Centers over RDMA-enabled Networks. In Proc. of the 6th IEEE/ACM International Symposium on Cluster Computing and the Grid, Singapore, May 2006.

[19] J. S. Plank, K. Li and M. Puening. Diskless Checkpointing. IEEE Transactions on Parallel and Distributed Systems, 9(10), pp. 972-986, October 1998.

[20] R. T. Mills. Dynamic adaptation to cpu and memory load in scientific applications. $\mathrm{PhD}$ thesis, Department of Computer Science, College of William and Mary, Fall, 2004.
[21] R. T. Mills, A. Stathopoulos, and D. S. Nikolopoulos. Adapting to memory pressure from within scientific applications on multiprogrammed COWs. In International Parallel and Distributed Processing Symposium (IPDPS 2004), Santq Fe, NM, USA, 2004.

[22] R. T. Mills, A. Stathopoulos, and E. Smirni. Algorithmic modifications to the Jacobi-Davidson parallel eigensolver to dynamically balance external CPU and memory load. In 2001 International Conference on Supercomputing, pages 454-463. ACM Press, 2001.

[23] J. Nieplocha, M. Krishnan, B. Palmer, V. Tipparaju, and Y. Zhang. Exploiting processor groups to extend scalability of the ga shared memory programming model. In $A C M$ Computing Frontiers, Italy, 2005.

[24] D. Nikolopoulos. Malleable Memory Mapping: UserLevel Control of Memory Bounds for Effective Program Adaptation. In Proc. of the 17th IEEE/ACM International Parallel and Distributed Processing Symposium (IPDPS'03), Nice, France, Apr. 2003.

[25] D. Nikolopoulos and C. Polychronopoulos. Adaptive Scheduling under Memory Pressure on Multiprogrammed Clusters. In Proc. of the 2nd IEEE/ACM International Conference on Cluster Computing and the Grid (ccGrid'02), pages 22-29, Berlin, Germany, May 2002.

[26] J. Oleszkiewicz, L. Xiao, and Y. Liu. Parallel network ram: Effectively utilizing global cluster memory for large data-intensive parallel programs. In 2004 International Conference on Parallel Processing (ICPP'2004), pages 353-360, 2004.

[27] H. Pang, M. J. Carey, and M. Livny. Memory-adaptive external sorting. In R. Agrawal, S. Baker, and D. A. Bell, editors, 19th International Conference on Very Large Data Bases, August 24-27, 1993, Dublin, Ireland, Proceedings, pages 618-629. Morgan Kaufmann, 1993.

[28] Y. Saad. SPARSKIT: A basic toolkit for sparse matrix computations. Technical Report 90-20, Research Institute for Advanced Computer Science, NASA Ames Research Center, Moffet Field, CA, 1990. Software currently available at $<$ ftp://ftp.cs.umn.edu/dept/sparse/ $>$.

[29] A. Stathopoulos, S. Öğüt, Y. Saad, J. R. Chelikowsky, and H. Kim. Parallel methods and tools for predicting material properties. Computing in Science and Engineering, 2(4):19-32, 2000.

[30] S. Vadhiyar and J. Dongarra. A Performance Oriented Migration Framework for the Grid. Technical Report, Innovative Computing Laboratory, University of Tennessee, Knoxville, 2002.

[31] G. Voelker, E. Anderson, T. Kimbrel, M. Feeley, J. Chase, A. Karlin, and H. Levy. Implementing Cooperative Prefetching and Caching in a Globally-Managed Memory System. pages 33-43, Madison, Wisconsin, June 1999.

[32] P. R. Woodward, S. Anderson, D. H. Porter and A. Iyer. Cluster Computing in the SHMOD Framework on the NSF TeraGrid. Technical Report, Laboratory for Computational Science and Engineering, University of Minnesota, April 2004. 